\title{
Chapter 13 \\ Disruption of Pollination Services by Invasive Pollinator Species
}

\author{
Carolina L. Morales, Agustín Sáez, Lucas A. Garibaldi, and Marcelo A. Aizen
}

\begin{abstract}
Plant-pollinator interactions and associated pollination services are essential for crop production and the integrity of terrestrial ecosystem services. Introduced pollinators, in particular social bees such as honeybees and bumblebees, have become invaders in many regions of the world, strongly affecting the pollination of native, cultivated, and non-native plants. These effects can be direct, when invaders interact with local flowering plants, or indirect, when invaders modify the interaction of native pollinators with flowering plants. Direct effects on pollination depend on whether the plant benefits from the flower visits are greater than their costs, a relationship that can be density dependent. Shifts from mutualism to antagonism occur when invasive pollinators reach extremely high densities, because the interaction costs exceed the benefits. Indirect effects depend on whether pollinator invaders alter the benefit-cost ratio of native pollinator visits, displace them, or trigger reductions in native pollinator diversity. Through a literature review, we found that the impacts of invasive pollinators on pollination were predominantly negative for native plants, mixed for crops, and positive for invasive plants. Furthermore, they can synergistically interact with other stressors on pollination such as climate change and habitat disturbance. Although invasive pollinators can back up pollination of some native plants in highly disturbed habitats, and some crops in intensively modified agro-ecosystems, they cannot replace the role of a diverse pollinator assemblage for wild plant reproduction and crop yield. Hence, managing agro-ecosystems for enhancing wild pollinator diversity, and avoiding further introductions of non-native pollinators, are realistic cost-effective measures for the provision and stability of pollination services.
\end{abstract}

\footnotetext{
C.L. Morales $(\varangle) \bullet A$. Sáez $\bullet$ M.A. Aizen

Laboratorio Ecotono, INIBIOMA (CONICET-Universidad Nacional del Comahue),

Bariloche, Río Negro, Argentina

e-mail: moralesc@comahue-conicet.gob.ar; agustinsaezmail@gmail.com;

maizen@comahue-conicet.gob.ar
}

L.A. Garibaldi

Grupo de Investigación en Agroecología (AGRECO), Sede Andina, Universidad Nacional de Río Negro (UNRN) and CONICET, Bariloche, Río Negro, Argentina

e-mail: lgaribaldi@unrn.edu.ar 
Keywords Apis $\bullet$ Bombus $\bullet$ Climate change $\bullet$ Crop $\bullet$ Disturbance $\bullet$ Indirect effects - Introduced pollinators $\bullet$ Mutualism

\subsection{Introduction}

Pollination services contribute directly and indirectly to human welfare. Biotic pollination, the transfer of pollen between flowers by free-living animals, contributes to the sexual reproduction of approximately $90 \%$ of the flowering plants (Angiosperma) (Ollerton et al. 2011) and increases fruit or seed quality or quantity of about $70 \%$ of the major crops worldwide (Klein et al. 2007). Furthermore, $5 \%$ to $8 \%$ of the global crop production for human consumption can be lost directly in the absence of animal pollination (Aizen et al. 2009), and many crops, from which vegetative parts are harvested, rely on pollinators for breeding and seed production. Indeed, animal pollination is a key ecosystem service, with plant-pollinator interactions having a significant role in maintaining the integrity of most terrestrial ecosystems, indirectly allowing the delivery of other ecosystem services such as primary production and carbon sequestration.

Invasive species are one of the main drivers of anthropogenic global change. As such, they can substantially disrupt the capacity of ecosystems to deliver services, including pollination (Traveset and Richardson 2006; Schweiger et al. 2010). Many bee species of diverse genera have become established in the wild out of their ranges as a consequence of accidental or intentional introductions (Goulson 2003; Bartomeus et al. 2013). For instance, some cavity-nesting species have been accidentally transported together with their host materials (e.g., Anthidium manicatum; Gibbs and Sheffield 2009), whereas other cavity-nesting species (e.g., Megachile and Osmia), as well as social or colonial genera Apis and Bombus, have been intentionally introduced for pollination purposes (Goulson 2003; Gibbs and Sheffield 2009; Schweiger et al. 2010). However, only a small fraction of these introduced species became "invasive species", that is, with individuals dispersing, surviving, and reproducing at multiple sites across a greater or lesser spectrum of habitats and extent of occurrence (Blackburn et al. 2011). Moreover, according to a literature survey that identified 403 primary research publications that investigated the ecological effects of invasive insects (Kenis et al. 2008), only 4 of 72 species were pollinators (Megachile rotundata, M. apicalis, Apis mellifera, Bombus terrestris), with only A. mellifera and $B$. terrestris demonstrating ecological impacts in the field (cf. the impact of an invasive solitary orchid bee on an invasive plant reproduction) (Table 13.1).

The massive and unquestioning active introduction of honeybee, A. mellifera hives is still occurring, whereas that of bumblebee colonies (Bombus spp., in particular B. terrestris) is just beginning (Fig. 13.1). Both introduced bumblebees as well as African honeybees (Box 13.1) continue to expand their ranges in the Americas at surprisingly high rates. Therefore, a comprehensive revision of the consequences of these pollinator invasions on pollination services is both timely and necessary. 


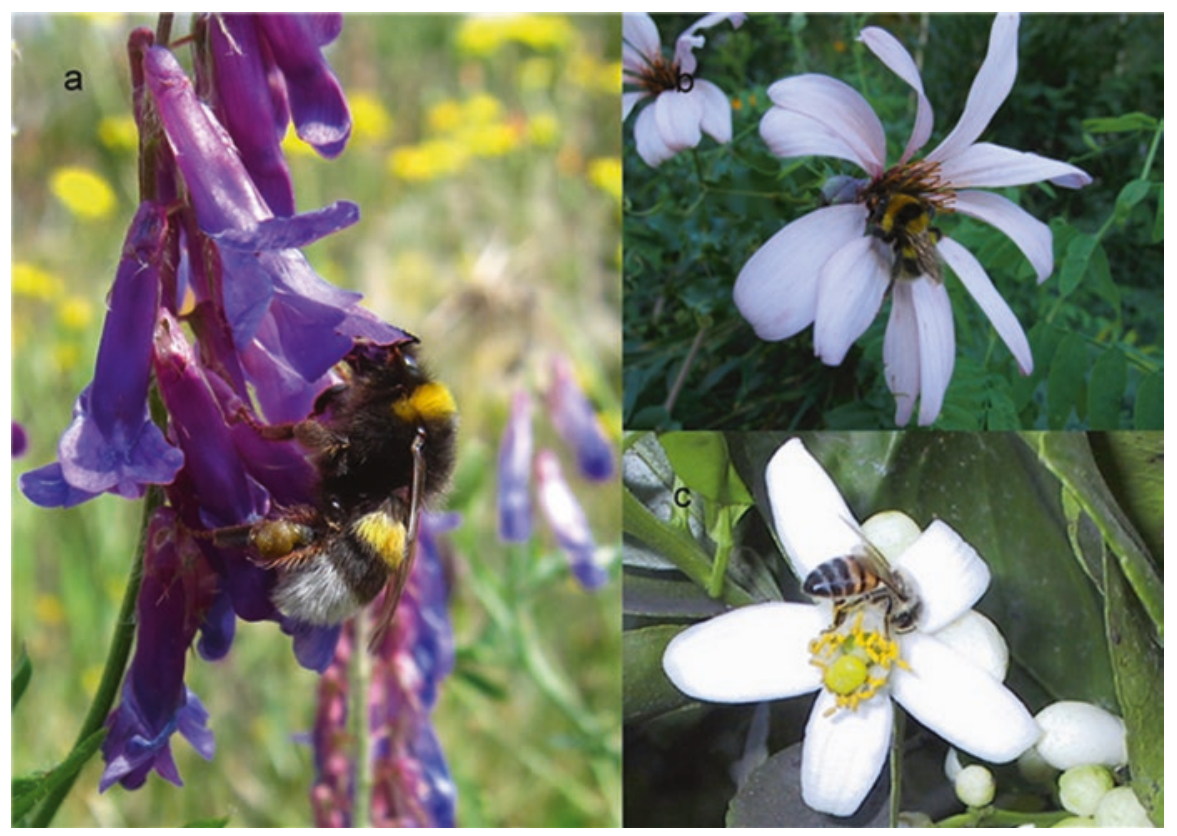

Fig. 13.1 Introduced pollinators that have become invasive in different parts of the world: Bombus terrestris (a), Bombus ruderatus (b), and Apis mellifera (c) (Photograph by Agustín Saez (a), Carolina L. Morales (b), and Natacha Chacoff (c))

\section{Box 13.1: Invasion History of Honeybees in the Americas}

The natural range of the western honeybee, Apis mellifera, extends from northern Europe to southern Africa, and from the British Isles to the Ural Mountains, western Iran, and the Arabian peninsula. More than 25 subspecies of honeybees are recognized. The African honeybee, the subspecies A. mellifera scutellata, originally occurred in southern Africa and was first introduced to Brazil in the 1950s with the aim to establish honeybee populations better adapted to tropical conditions. Since introduction, this subspecies has spread through tropical and subtropical Americas, from northern Argentina to southwestern USA, being considered one of the most successful cases of biological invasions (Schneider et al. 2004).

The African honeybee initially interbred with other European honeybee subspecies, such as the Italian A. m. ligustica and the Iberian A. m. iberiensis, also introduced in America, producing the hybrid commonly known as "Africanized honeybee." However, although substantial hybridization occurs when African honeybees invade areas with populations of European 


\section{Box 13.1 (continued)}

subspecies, European characteristics tend to be lost over time, and through much of its invaded range in the New World, African characteristics have been preserved (Schneider et al. 2004). The terminology used to name and describe the descendants of A. mellifera scutellata in the New World is highly controversial. In this chapter we refer to them as "African honeybees" and use the term "feral" to refer to populations established in the wild, in opposition to "managed" for those being kept in bee hives.

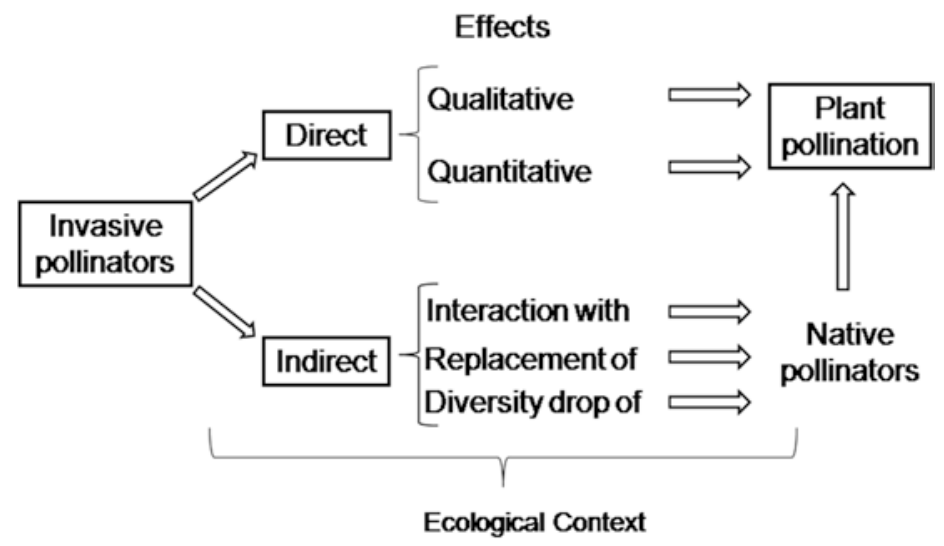

Fig. 13.2 Diagram representing the direct and indirect effects of invasive pollinators on plant pollination

This chapter reviews the impacts of introduced invasive pollinators in the broad sense on flower-visiting animals, and on native pollinators and pollination services. The conceptual framework we present includes direct and indirect effects, wherein direct effects are characterised by the benefit-cost balance of the interaction between the invasive pollinator and a given plant, which in turn takes into account its per capita (qualitative) effect and its mass (quantitative) effect (Fig. 13.2). Indirect effects occur additionally when this novel pollinator impacts the pollination of a given plant, either by their interaction with the native flower visitors, by replacing efficient pollinators, or by driving reductions in pollinator diversity. These effects will ultimately be strongly influenced by the ecological and community context, including their interaction with other environmental stressors such as climate change, habitat disturbance, and invasive plants (Fig. 13.2). The main mechanisms behind these impacts are illustrated in this review, using examples of diverse taxa of native, cultivated, and invasive plants from different ecosystems worldwide (Table 13.1. 


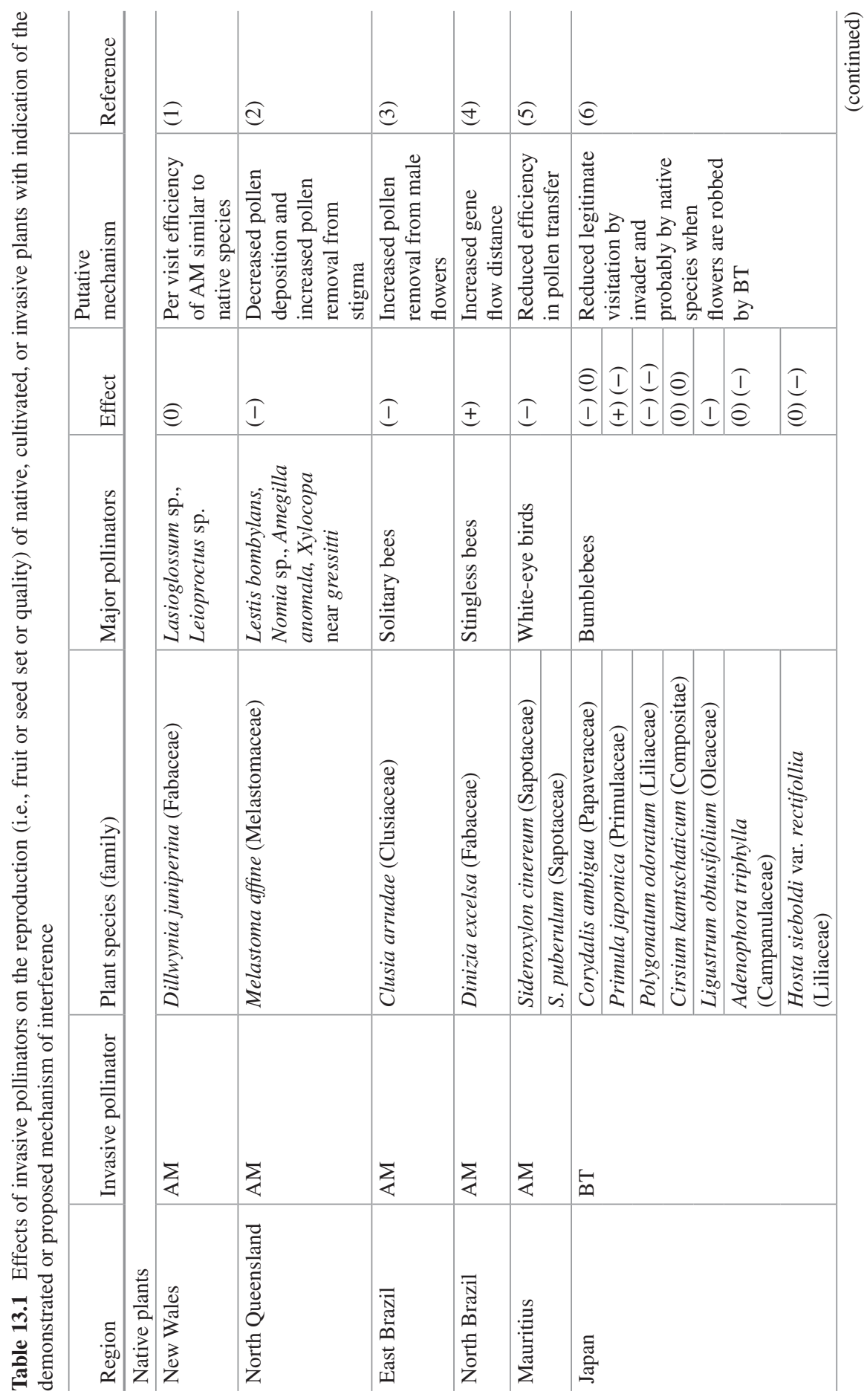




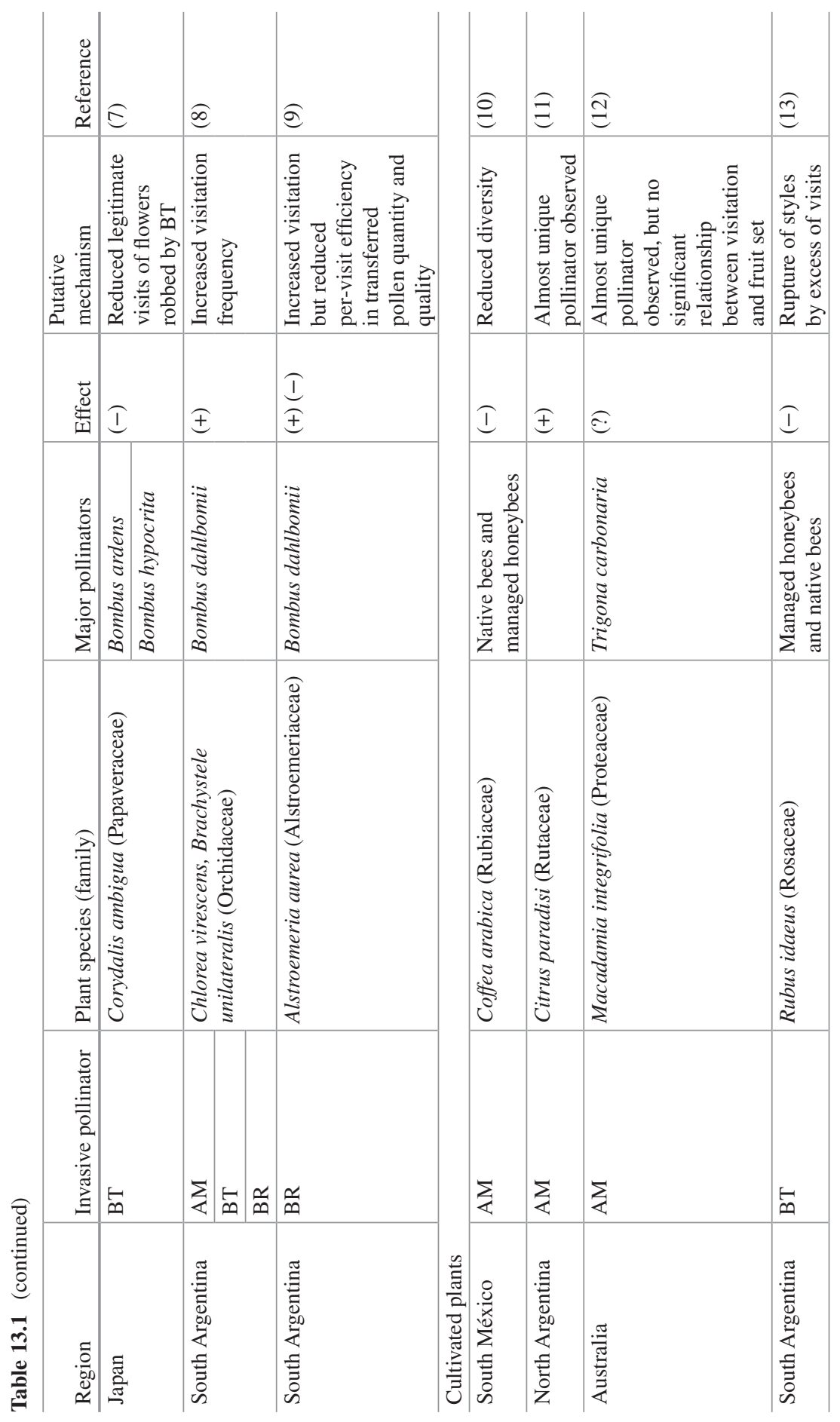




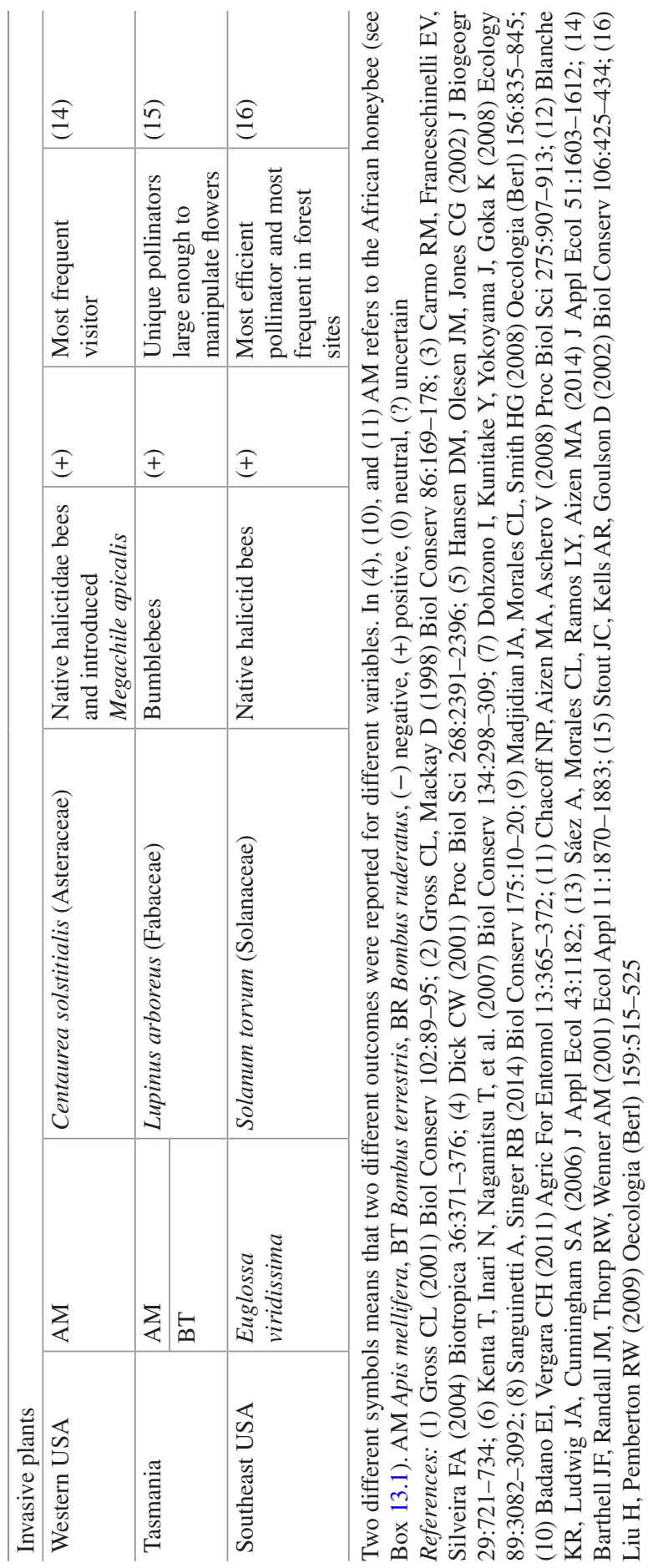




\subsection{Direct Effects of Invasive Pollinators on Plant Pollination}

\subsubsection{Per Capita Effect}

Plant-pollinator interaction can be viewed as a benefit-cost relationship, which can be mutualistic or antagonistic, depending on whether the net benefit (benefit minus cost) is positive or negative (Aizen et al. 2014, and references therein). From the perspective of a plant, the benefit obtained from a single flower visit is the transfer of high-quality conspecific pollen from anthers to stigma, and the cost involves the removal of floral rewards (nectar and pollen), flower damage, and the transfer of pathogens, among other matters. Invasive pollinators can disrupt the pollination of native plants, if the per-visit costs exceed the benefits. Trait matching between interacting partners is expected to enhance benefits (pollen transfer) while minimizing costs (pollen wastage and flower damage) (Schleuning et al. 2015).

Experimental manipulation of the identity of flower visitors in isolated plants has been a powerful tool to estimate the net benefit provided by alternative pollinators. For example, in native Dillwyinia juniperiana, the probability of a flower setting fruit after a single visit did not significantly differ between honeybees and native bees; however, fruit set was $10 \%$ greater when native bees were the only visitors compared to honeybees (Table 13.1). In Japan, where Bombus terrestris has become a widespread invader, seven native bumblebee-pollinated plant species were experimentally exposed to different treatments, simulating different invasion scenarios where B. terrestris was absent ("native" treatment), coexisted with native bumblebees ("mixed" treatment), or completely replaced them ("non-native" treatment) (Table 13.1). Five of seven species experienced reduced fruit set, fruit quality, or both, but only one species experienced increased fruit quality in the non-native versus native treatment. Visits by $B$. terrestris were of lower effectiveness (i.e., fruits per visit) than those of the native bumblebee because of physical mismatching between the length of the $B$. terrestris tongue and the length of the native plant corollas. Therefore, the per capita effect of a new interacting partner (invasive pollinator) on a plant pollination can vary according to the degree of matching between pollinator and plant traits.

\subsubsection{Mass Effect}

Invasive species typically reach much higher abundances than those observed in their native ranges or than their native counterparts in invaded regions (e.g., European and African honeybees; B. terrestris in South America and Japan and B. ruderatus in South America). These high abundances lead to unusually high visitation frequencies to flowers. As an example, Fig. 13.3 shows the distribution of visitation frequencies in eight plant-pollinator webs across different forest habitat types in southern Argentina, where only interactions with native pollinators are 


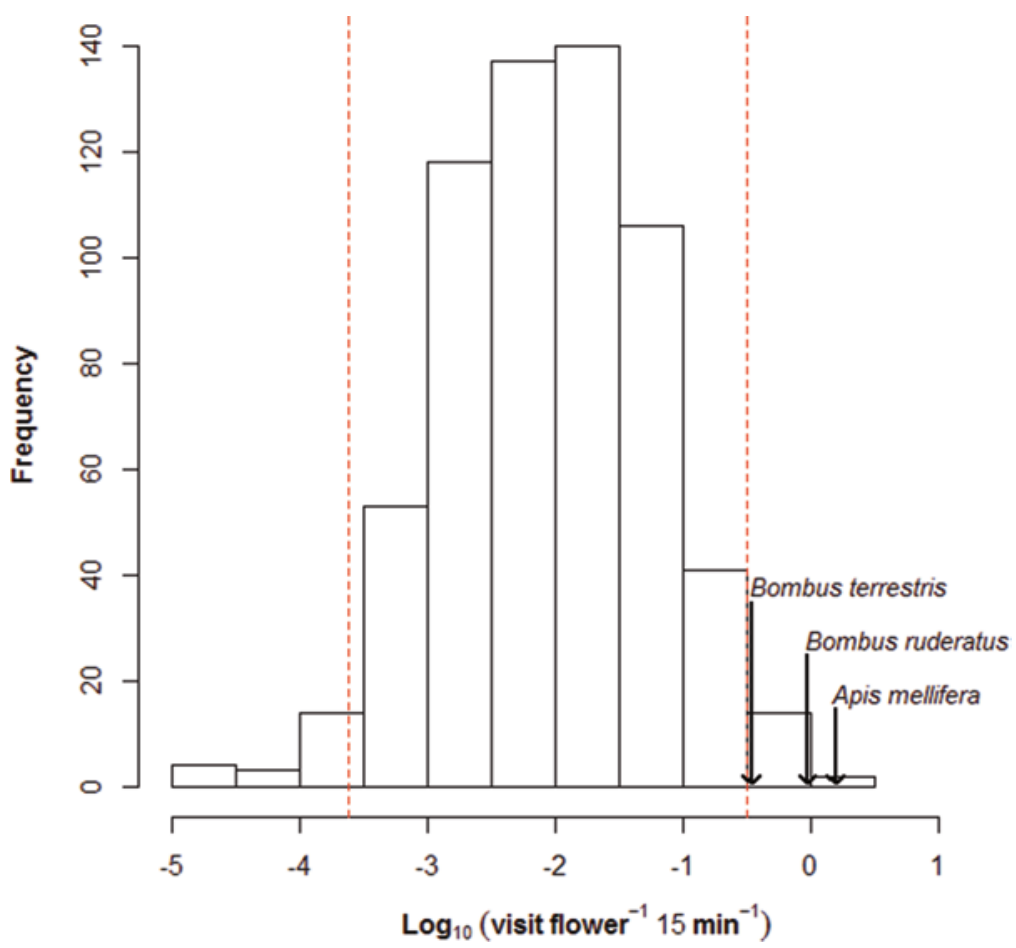

Fig. 13.3 Mean annual visitation rate of native pollinators on plants across eight forest habitat types in Southern Argentina. The dashed line represents the percentiles 2.5 and 97.5 of the frequency distribution. The arrows at the right side of the graph indicate the highest mean annual visitation rate achieved by the three invasive pollinator species in this area, namely, Bombus terrestris in Alstromeria aurea, B. ruderatus in Carduus nutans, and Apis mellifera in Cirsium vulgare (Data from Aizen et al. 2008, supplementary material, and Morales et al. 2013)

included. By contrast, the thick arrows indicate the maximum visitation frequency recorded for a plant-pollinator interaction in which invasive pollinators are involved. This histogram clearly illustrates how invasive pollinators tend to participate in interaction frequencies that are higher than "normal".

According to the benefit-cost conceptual model proposed by Aizen et al. (2014), benefits of the plant-flower visitor interactions increase asymptotically with interaction frequency while costs increase linearly (Fig. 13.4). Hence, from the perspective of the plant, a shift to antagonism from an otherwise mutualistic plant-animal interaction is especially prevalent when partners have disparate relative densities (Aizen et al. 2014). Therefore, the impact of this increase in interaction frequency will depend on whether, within the range of frequencies observed, interaction costs exceed their benefits.

Following the Aizen et al. (2014) conceptual model, Fig. 13.4 shows the expected density-dependent benefit-cost relationship between a plant species and two pollinator species that differ both in their interaction costs (straight lines) and their benefits (asymptotic curves). At any frequency, interactions with invasive pollinators 


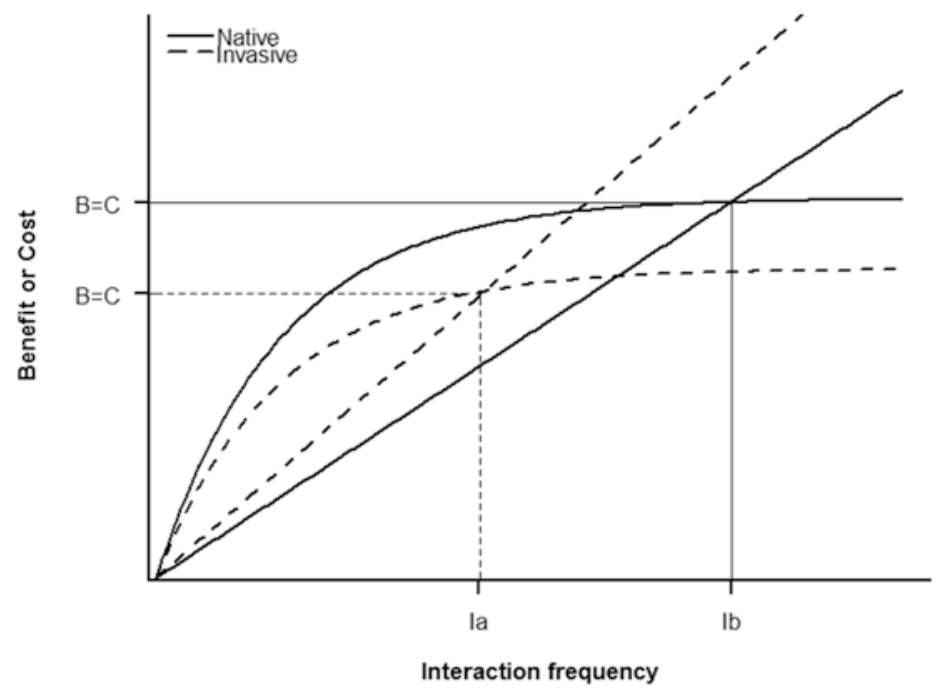

Fig. 13.4 Plant benefits $(B)$ and costs $(C)$ of increasing interaction frequency $(I)$ from native (solid lines) or invasive (dashed lines) pollinators. Benefits (e.g., seed set) increase nonlinearly and are expected to be greater from native than invasive pollinator visits, whereas costs (e.g., flower damage) increase linearly and are expected to be lower from native pollinator visits. Invasive pollinators are mutualists when $I<\mathrm{I} a$ (benefit $>$ cost) and antagonists when $I>I a$ (benefit $<$ cost). Native pollinators are mutualists when $I<I b$ and antagonists when $I>I b$. If invasive pollinators become too abundant, they are expected to behave mostly as antagonists from the perspective of the plant

that provide lower benefits and entail higher costs than interactions with the average native pollinator are expected to result in a lower net benefit. Moreover, mutualism is expected to switch into antagonism at a lower interaction frequency when the plant interacts with an invasive pollinator (Ia) than with an average native pollinator $(I b)$. Conversely, when the abundance of floral resources is high relative to visitors, the density of invasive flower visitors is expected to be within the range of increasing net benefits (Aizen et al. 2014). This situation frequently occurs during short periods in agricultural landscapes when mass flowering crops provide a high abundance of floral resources. In such cases, increases in visitation frequency are expected to benefit yields in pollen-limited crops.

In tropical America, the invasion of feral African honeybees seems to have augmented pollination and boosted the yields of coffee, Coffea arabica, as suggested by the substantial increase in coffee yield in many neotropical countries. This increase has been partly coincident with the establishment of African honeybees in the region (Roubik 2002, and references therein). However, in the same crop, fruit set was negatively related to African honeybee visitation frequency (Table 13.1; Fig. 13.5a). This result is consistent with a shift in the balance from mutualism to antagonism at excessive visitation frequencies, given that the total number of honeybee foragers per flower appears to be sufficiently high to cause the rapid depletion of floral resources (but see also Sect. 13.3.3). In a temperate crop, an antagonist effect 

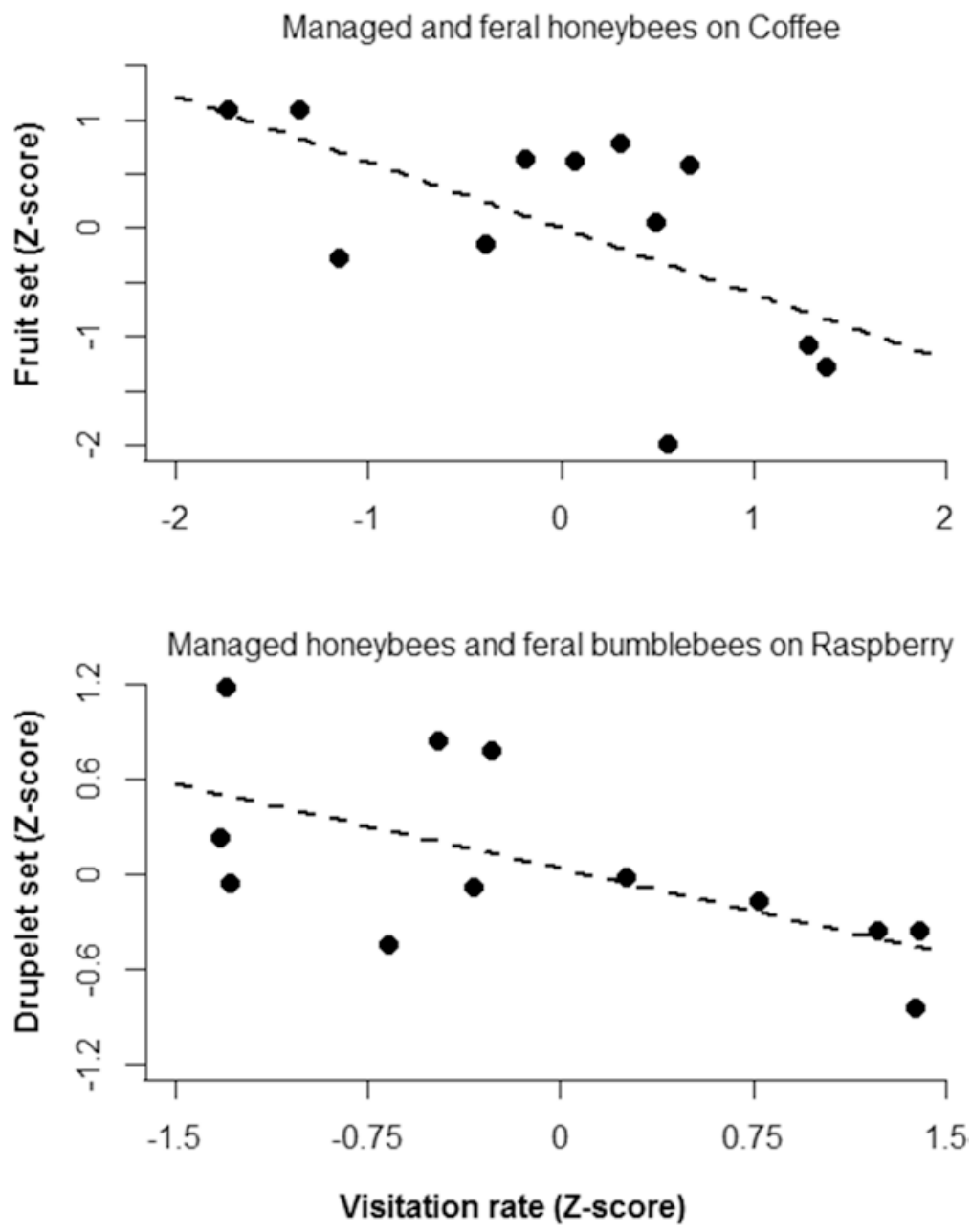

Fig. 13.5 Negative effect of increasing visitation rate of introduced pollinators (both managed and feral) on pollination services in two crop systems: fruit set of highland coffee, Coffea arabica, in tropical Southern Mexico (a) and drupelet set in raspberry, Rubus idaeus, in temperate Southern Argentina (b) (Data of coffee in $z$-score values were obtained from Garibaldi et al. (2013) supplementary material, original data from study (10) quoted in Table 13.1; data of raspberry from study (13) quoted in Table 13.1)

of increasing visitation frequency has resulted from a combination of managed honeybees and invasive $B$. terrestris (Table 13.1; Fig. 13.5b), whereas in raspberry fields in Southern Argentina, the exceptionally high abundance reached by $B$. terrestris has translated into visitation frequencies that both saturate benefits (pollen deposition) and increase interaction costs (style damage), thereby shifting the interaction from mutualism towards the antagonism threshold (Aizen et al. 2014). 


\subsection{Indirect Effects}

\subsubsection{Impacts Mediated by the Interaction with Native Pollinators}

Invasive pollinators can disrupt the pollination of plants by altering the outcome of interactions between plants and native pollinators. This disruption occurs through the modification of the behaviour of the native pollinators by the invasive pollinators. The most obvious mechanism for this is the reduction of visitation by native pollinators in the presence of invaders. For instance, in Mauritius, white-eye birds stop foraging on native Sapotaceae trees when honeybees deplete nectar (Table 13.1). In a Japanese bumblebee experiment, five plant species were exposed to a "mixed" treatment in addition to "non-native" and "native" treatments: the "mixed" treatment caused reduced fruit set and quality for Primula odorata, and increased fruit set but reduced fruit quality for Polygonium japonica, relative to the "native" treatment (Table 13.1). Thus, in $P$. odorata, reduced visitation by native bumblebees in the "mixed" compared to the "native" treatment suggests that the presence of B. terrestris disturbs native bumblebees, which may partly explain reproductive failure, given that legitimate visits is the primary factor explaining reproductive success.

A more subtle mechanism for this interaction is the modification of the costs or benefits provided to plants by native pollinators in the presence of invasive counterparts. For example, in North Queensland (Australia) honeybees not only deposited less pollen per visit than native bees in flowers of the shrub Melastoma affine (Table 13.1), but also removed pollen from stigmas previously deposited by native species, which reduces plant reproductive success. In Brazil, pollen-collecting honeybees visiting the native shrub Clusia arrudae removed almost all pollen from the male flowers (Table 13.1), leading native solitary bees visiting male flowers previously visited by honeybees to carry on their bodies less than $0.1 \%$ of the pollen grains carried by bees leaving flowers not visited by the honeybee. As a consequence, fruit set was negatively correlated with honeybee visitation to male flowers.

These examples show that the impacts of invasive pollinators can also be mediated by their interactions with native pollinators, triggering changes in the benefits and costs per visit of native pollinators or in the frequency of their visits. Therefore, single-visit experiments in isolated flowers are poor predictors of the overall impacts of invasive pollinators and should be complemented with other experimental and observational approaches.

\subsubsection{Impacts Caused by the Replacement of More Efficient Pollinators}

Invasive pollinators can outcompete native pollinators through competition for resources, nesting sites, or transmission of pathogens, among other mechanisms (Goulson 2003, and references therein). In relatively specialised plant pollination 
systems, this can lead to an almost complete replacement of major pollinators by invasive pollinators, whose impact would depend on the relative benefit-cost of the interaction of the invasive pollinator compared to the native pollinator with a given plant (Sects. 13.2.1 and 13.2.2). For instance, the reduced fruit set or fruit quality suffered by five of seven plants experimentally exposed to $B$. terrestris compared to native bumblebees (Table 13.1; Sect. 13.2.1) suggests that the ongoing replacement of native bumblebees by invasive $B$. terrestris can negatively affect the reproduction of native plants.

However, the replacement of major pollinators by invasive pollinators does not always have deleterious effects on the reproduction of native plants, particularly when visits by the major pollinators are too infrequent to fulfil pollination requirements. For instance, non-autogamous deceptive orchids strongly rely on a few specialist pollinator species for their reproduction. Chlorea virescens and Brachystele unilateralis, two orchids endemic to Southern Argentina and Chile, are unable to set seeds in the absence of pollinators. A 2-year study carried out after a B. terrestris invasion, coincidental with the almost functional local extinction of their putative pollinator, B. dahlbomii, revealed that, because their flowers were so large, none of the small remaining native pollinators were capable of transferring pollinaria to them (Table 13.1). In addition, invasive B. terrestris, B. ruderatus, and Apis mellifera were responsible for almost all the pollination events observed. Therefore, the unusually high fruiting success in the study area, compared to that reported for other temperate orchid species, might be attributed to the high incidence and visitation frequency of these invasive species (see Sect.13.2.2; Table 13.1).

In the same region, the per-visit benefit provided by invasive $B$. ruderatus to Alstroemeria aurea flowers are lower compared to native $B$. dahlbomii because the former deposits less and lower quality pollen than the native species. However, this reduced per-visit efficiency of the invasive species seems to be at least partially compensated by visitation frequencies higher than those historically recorded for the native species (Morales et al. 2013, Table 13.1).

\subsubsection{Impacts of Invasive Pollinators Mediated by Reductions on Native Flower Visitor Diversity}

The effects of pollinator species diversity on pollination services are expected to be mediated by changes in pollinator functional diversity, that is, the among-species variation in behavioural, morphological, and physiological traits relevant to pollination function (Fründ et al. 2013; Gagic et al. 2015). Functional diversity of pollinator assemblages increases the level and stability of pollination services through several non exclusive mechanisms (Tscharntke et al. 2005). First, functional richness and evenness, two components of functional diversity, should enhance niche complementarity, such as differences among flower visitors in temperature preferences or activity periods (Fründ et al. 2013; see also Sects. 13.4.1 and 13.4.2). 
Second, functional diversity can enhance pollination services because of "sampling effects", that is, higher diversity of traits associated with higher species diversity increases the probability of finding a particular pollinator species, characterised by a series of traits, which increases the pollination efficiency of one or several particular plant species (Tscharntke et al. 2005; Schleuning et al. 2015). Third, functional diversity can enhance particular interspecific interactions. For example, in almond orchards in the USA, the presence of non-Apis bees changed the foraging behaviour of honeybees, which tended to fly more often between tree rows, increasing the pollination effectiveness of a single honeybee visit, resulting in greater fruit set than in orchards where non-Apis bees were absent (Brittain et al. 2013). Therefore, pollinator diversity can synergistically increase pollination service through species interactions that alter the behaviour and resulting functional quality of a dominant pollinator species (Brittain et al. 2013). Last, pollinator diversity increases the stability of pollination services even when different pollinators are functionally equivalent because it buffers the demographic vagaries of individual species.

A recent synthesis of pollinator and fruit set surveys from 600 fields involving 41 crop systems revealed that pollinator richness had a positive and significant effect on wild pollinator visitation, which in turn consistently enhanced fruit set in all crop systems without exception. In contrast, honeybee visitation increased fruit set significantly in only $14 \%$ of them (Garibaldi et al. 2013). This finding confirms the global positive effect of pollinator diversity on pollination services.

By displacing native pollinators, invasive pollinators can reduce pollinator diversity. Evidence of such reductions in the diversity of native pollinators resulting from pollinator invasions is mixed, and the cause-effect relationship between the phenomena is often hard to separate from other confounding factors (Sect. 13.4). However, negative effects on native pollinator diversity prevail when invaders become too abundant. For instance, in Tasmania, Goulson et al. (2002) found no effect of B. terrestris on native pollinator diversity, but a negative effect of $A$. mellifera, which was by far the most abundant bee species.

Evidences of a shortage of pollination service driven by decreased pollinator diversity as a result of pollinator invasions are even scarcer. In coffee plantations in Mexico, the number of honeybee workers per plantation was negatively correlated with native pollinator diversity and fruit set (Table 13.1; Fig. 13.5a). Although the generality of this type of impact of invasive species should be tested, the foregoing examples illustrate the potential consequences of losses of biodiversity on pollination services.

\subsection{Interaction of Invasive Pollinators and Other Drivers of Global Change}

Pollination services are under the threat of many stressors, which rarely act in isolation (Schweiger et al. 2010). Rather, they can interact in complex and even nonadditive manners, either modulating or amplifying their individual impacts (González-Varo 
et al. 2013). Climate change, habitat disturbance, and plant invasions are among these environmental stressors that can interact with invasive pollinators.

\subsubsection{Climate Change}

The impact of invasive pollinators is influenced by climate change. Apis mellifera and Bombus terrestris are social and generalist species with broad resource and habitat niches, long foraging seasons, and a widespread distribution within their regions of origin. Therefore, they are expected to be less sensitive to global warming per se and concomitant phenological or spatial mismatches than most native pollinators, which are predominantly solitary and on average more specialised (Schweiger et al. 2010). A recent review by Forrest (2015) concludes that, although primarily observational studies suggest that phenologies of co-occurring plants and pollinators tend to respond similarly to the same environmental cue, some interacting species can suffer phenological mismatches. However, the impact on plant reproduction is unknown, largely because of a lack of research.

Invasive pollinators can complement the pollination service provided by native pollinators under increasing temperature and precipitation variability (see Sect. 13.3.3), or compensate for the plant-pollinator mismatches resulting from the spatial or phenological shifts experienced by native pollinators, thus benefitting generalist plants. On the other hand, they are expected to exacerbate the detrimental effect of climate change on highly specialist plant-pollinator systems by increasing the relative competitive ability of invasive over more efficient native pollinators (Sect. 13.3.2).

\subsubsection{Habitat Disturbance}

Habitat alteration and invasive pollinators can affect native pollinators to the same magnitude by decreasing visitation rates (Montero-Castaño and Vilà 2012). Moreover, invasive pollinators are usually associated with disturbed habitats (Aizen et al. 2008, and references therein), where, given the correlative nature of most ecological studies, the collapse of native pollinators can hardly be attributed to either invasive species or habitat disturbance per se. A possible interpretation of these patterns is that invasive pollinators take advantage of habitat disturbance, becoming more successful invaders and therefore competitively superior to native counterparts that are actively displaced by invaders in disturbed habitats. Therefore, habitat disturbance is expected to exacerbate the negative impacts of invasive pollinators on native pollinators. Alternatively, native pollinators might decline as a direct consequence of habitat disturbance, being passively replaced by invasive pollinators that take advantage of niche release. Regardless of the cause-effect relationship, the 
consequences for pollination services will depend on whether invasive pollinators can compensate for the loss of native pollinators in disturbed habitats. In the Chaco Dry Forest of Argentina, the consistently higher visitation frequency of feral honeybees on plants growing in small forest fragments cannot fully compensate for the loss of native pollinators, but it does seem to ameliorate the magnitude of the negative effects of fragmentation on reproduction of wild plants (Aguilar et al. 2006, and references therein). In the extreme, invasive pollinators can overcompensate for the loss of native pollinators. For instance, in Amazonian rainforests honeybees are the unique pollinators able to fly distances long enough to visit isolated populations of the tropical tree species, Dinizia excelsa, that remain in pastures and forest fragments. The trees in these isolated fragments produce higher seed yields than trees growing in the forest because honeybees transported pollen from more distant parents than native pollinators (Table 13.1).

The intensification of agricultural systems is also a strong driver of native pollinator losses (Garibaldi et al. 2014). Various syntheses revealed that the diversity of native pollinators and their abundance in crops negatively correlates with increasing distances from crop borders and decreasing areas of natural and seminatural habitats in the local landscape. Although the detrimental effect on pollination services has also been confirmed (Garibaldi et al. 2014), these impacts seem negligible when feral honeybees are the major pollinators (Table 13.1).

In Australia, stingless bees are important pollinators of macadamia, Macadamia integrifolia, but the honeybee has been reported as the almost only pollinator of this crop. This finding was thought to be a result of a potentially higher resistance to pesticides in honeybees compared to the native bees (Table 13.1). Thus, invasive pollinators can back up pollination in extremely disturbed habitats, where the native pollinators are disadvantaged. In any case, an obvious conclusion is that, to support healthy and rich pollinator assemblages on which a diversified and stable pollination service relies, habitat disturbance should be avoided or minimized, and agricultural practices that mitigate the impact of increasing land use intensification should be employed (Garibaldi et al. 2014).

\subsubsection{Invasive Plants}

The invasion of introduced flowering plants is often pollen limited and, although they can rapidly integrate into local plant-pollinator webs through interactions with native pollinators (Memmott and Waser 2002), when invasive pollinators are present introduced plants tend to interact more frequently with them beyond the effects of habitat disturbance (Aizen et al. 2008).

Differential interactions between invasive plants and pollinators may arise from the fact that invasive pollinators tend to be more generalist than most native pollinators (Memmott and Waser 2002; Aizen et al. 2008), or from a better morphological matching, particularly in large or highly specialised flowers. For instance, in Tasmania native bees are too small to manipulate flowers of the invasive Lupinus 
arboreaus, which rely on $A$. mellifera and B. terrestris to set seeds (Table 13.1). Similarly, the honeybee also promotes seed set of Centaurea solticialis in the USA, because it is larger in size compared to most native bees (Table 13.1). This difference can lead to an "invasional meltdown" (sensu Simberloff and Von Holle 1999), increasing the invasive potential of the interacting partners, or exacerbating their individual impacts (Aizen et al. 2008, and references therein).

\subsection{Conclusions}

Pollination services enhance the sexual reproduction of most flowering plants involved in many terrestrial ecosystem services, and are essential to guarantee diverse and abundant high-quality crop yields, which all together contribute to human well-being. Paradoxically, invasive pollinators can threaten the pollination service for which most of them were primarily introduced. In highly disturbed or intensively managed habitats, where wild and cultivated plants might suffer high pollination limitation, invasive pollinators might ameliorate such a pollination deficit. However, they cannot replace the function of a diverse assemblage of native pollinators. Therefore, avoiding further introductions, as well as managing and restoring agro-ecosystems to improve the habitat conditions needed by wild pollinators to survive (Garibaldi et al. 2014), emerge as the highest priorities to guarantee healthy and long-term pollination services.

Acknowledgments We thank N. Bartomeus and M. Vilà for helpful comments on a previous draft. We also thank PICT 2012-3015 funding. A.S. holds a doctoral scholarship from CONICET.

\section{References}

Aguilar R, Ashworth L, Galetto L et al (2006) Plant reproductive susceptibility to habitat fragmentation: review and synthesis through a meta-analysis. Ecol Lett 9:968-980

Aizen MA, Morales CL, Morales JM (2008) Invasive mutualists erode native pollination webs. PLoS Biol 6:0396-0403

Aizen MA, Garibaldi LA, Cunningham SA et al (2009) How much does agriculture depend on pollinators? Lessons from long-term trends in crop production. Ann Bot 103:1579-1588

Aizen MA, Morales CL, Vázquez DP et al (2014) When mutualism goes bad: density-dependent impacts of introduced bees on plant reproduction. New Phytol 204:322-328

Bartomeus I, Ascher JS, Gibbs J et al (2013) Historical changes in northeastern US bee pollinators related to shared ecological traits. Proc Natl Acad Sci U S A 110:4656-4660

Blackburn TM, Pyšek P, Bacher S et al (2011) A proposed unified framework for biological invasions. Trends Ecol Evol 26:333-339

Brittain C, Williams N, Kremen C et al (2013) Synergistic effects of non-Apis bees and honeybees for pollination services. Proc Biol Sci 280:2012-2767

Forrest JRK (2015) Plant-pollinator interactions and phenological change: what can we learn about climate impacts from experiments and observations? Oikos 124:4-13 
Fründ J, Dormann CF, Holzschuh A et al (2013) Bee diversity effects on pollination depend on functional complementarity and niche shifts. Ecology 94:2042-2054

Gagic V, Bartomeus I, Jonsson T et al (2015) Functional identity and diversity of animals predict ecosystem functioning better than species-based indices. Proc $\mathrm{R}$ Soc $\mathrm{B}$ Biol Sci 282:2014-2620

Garibaldi LA, Steffan-Dewenter I, Winfree R et al (2013) Wild pollinators enhance fruit set of crops regardless of honey bee abundance. Science 339:1608-1611

Garibaldi LA, Carvalheiro LG, Leonhardt SD et al (2014) From research to action: enhancing crop yield through wild pollinators. Front Ecol Environ 12(8):439-447

Gibbs J, Sheffield CS (2009) Rapid range expansion of the wool-carder bee, Anthidium manicatum (Linnaeus) (Hymenoptera: Megachilidae), in North America. J Kansas Entomol Soc 82:21-29

González-Varo JP, Biesmeijer JC, Bommarco R et al (2013) Combined effects of global change pressures on animal-mediated pollination. Trends Ecol Evol 28:524-530

Goulson D (2003) Effects of introduced bees on native ecosystems. Annu Rev Ecol Evol Syst 34:1-26

Goulson D, Stout JC, Kells AR (2002) Do exotic bumblebees and honeybees compete with native flower-visiting insects in Tasmania? J Insect Conserv 6:179-189

Kenis M, Auger-Rozenberg M-A, Roques A et al (2008) Ecological effects of invasive alien insects. Biol Invasions 11:21-45

Klein A-M, Vaissière BE, Cane JH et al (2007) Importance of pollinators in changing landscapes for world crops. Proc Biol Sci 274:303-313

Memmott J, Waser NM (2002) Integration of alien plants into a native flower-pollinator visitation web. Proc Biol Sci 269:2395-2399

Montero-Castaño A, Vilà M (2012) Impact of landscape alteration and invasions on pollinators: a meta-analysis. J Ecol 100:884-893

Morales CL, Arbetman MP, Cameron SA et al (2013) Rapid ecological replacement of a native bumble bee by invasive species. Front Ecol Environ 11:529-534

Ollerton J, Winfree R, Tarrant S (2011) How many flowering plants are pollinated by animals? Oikos 120:321-326

Roubik DW (2002) The value of bees to the coffee harvest. Nature (Lond) 417:2002

Schleuning M, Fründ J, García D (2015) Predicting ecosystem functions from biodiversity and mutualistic networks: an extension of trait-based concepts to plant-animal interactions. Ecography 38:1-13

Schneider S, DeGrandi-Hoffman G, Smith DR (2004) The African honey bee: factors contributing to a successful biological invasion. Annu Rev Entomol 49:351-376

Schweiger O, Biesmeijer JC, Bommarco R et al (2010) Multiple stressors on biotic interactions: how climate change and alien species interact to affect pollination. Biol Rev 85:777-795

Simberloff D, Von Holle B (1999) Positive interactions of nonindigenous species: invasional meltdown ? Biol Invasions 1:21-32

Traveset A, Richardson DM (2006) Biological invasions as disruptors of plant reproductive mutualisms. Trends Ecol Evol 21:208-216

Tscharntke T, Klein AM, Kruess A (2005) Landscape perspectives on agricultural intensification and biodiversity-ecosystem service management. Ecol Lett 8:857-874 